\title{
An upper limit for the water outgassing rate of the main-belt comet 176P/LINEAR observed with Herschel/HIFI ${ }^{\star}$
}

\author{
M. de Val-Borro ${ }^{1, \star \star}$, L. Rezac ${ }^{1}$, P. Hartogh ${ }^{1}$, N. Biver ${ }^{2}$, D. Bockelée-Morvan ${ }^{2}$, J. Crovisier ${ }^{2}$, M. Küppers ${ }^{3}$, D. C. Lis ${ }^{4}$, \\ S. Szutowicz ${ }^{5}$, G. A. Blake ${ }^{4}$, M. Emprechtinger ${ }^{4}$, C. Jarchow ${ }^{1}$, E. Jehin ${ }^{6}$, M. Kidger ${ }^{7}$, L.-M. Lara ${ }^{8}$, E. Lellouch ${ }^{2}$, \\ R. Moreno ${ }^{2}$, and M. Rengel ${ }^{1}$
}

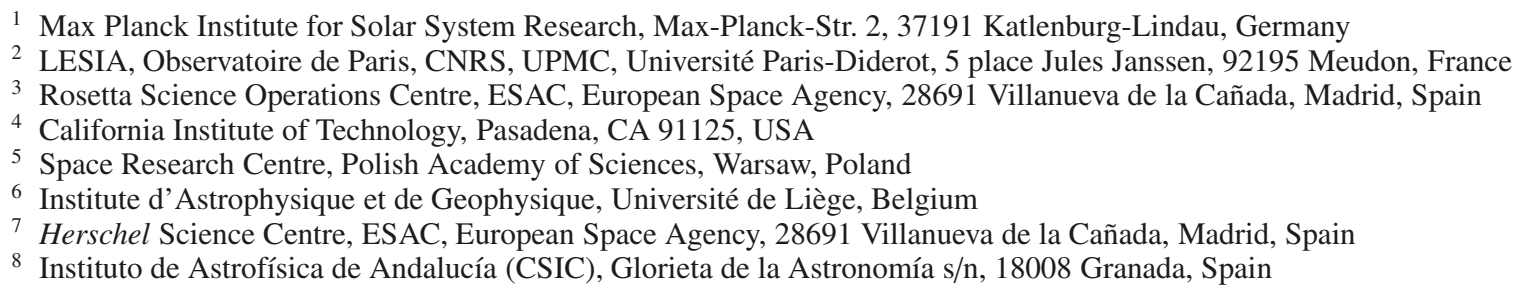

Received 5 August 2012 / Accepted 25 August 2012

\section{ABSTRACT}

\begin{abstract}
176P/LINEAR is a member of the new cometary class known as main-belt comets (MBCs). It displayed cometary activity shortly during its 2005 perihelion passage, which may be driven by the sublimation of subsurface ices. We have therefore searched for emission of the $\mathrm{H}_{2} \mathrm{O} 1_{10}-1_{01}$ ground state rotational line at $557 \mathrm{GHz}$ toward 176P/LINEAR with the Heterodyne Instrument for the Far Infrared (HIFI) onboard the Herschel Space Observatory on UT 8.78 August 2011, about 40 days after its most recent perihelion passage, when the object was at a heliocentric distance of $2.58 \mathrm{AU}$. No $\mathrm{H}_{2} \mathrm{O}$ line emission was detected in our observations, from which we derive sensitive 3- $\sigma$ upper limits for the water production rate and column density of $<4 \times 10^{25} \mathrm{~mol} \mathrm{~s}^{-1}$ and of $<3 \times 10^{10} \mathrm{~cm}^{-2}$, respectively. From the peak brightness measured during the object's active period in 2005, this upper limit is lower than predicted by the relation between production rates and visual magnitudes observed for a sample of comets at this heliocentric distance. Thus, 176P/LINEAR was most likely less active at the time of our observation than during its previous perihelion passage. The retrieved upper limit is lower than most values derived for the $\mathrm{H}_{2} \mathrm{O}$ production rate from the spectroscopic search for CN emission in MBCs.
\end{abstract}

Key words. comets: individual: 176P/LINEAR - submillimeter: planetary systems - techniques: spectroscopic

\section{Introduction}

Classical main-belt asteroids are small bodies that orbit the Sun in low inclination and low eccentricity orbits between the orbits of Mars and Jupiter. Physically, asteroids are thought to be devoid of volatiles, while comets are icy bodies that become active in the inner solar system thanks to the sublimation of ices, mostly water. Comets originate in the outskirts of the solar system beyond the snow line, where temperatures in the solar nebula were low enough for water to condense onto icy grains (Hayashi 1981). A new class of bodies has been discovered recently, the so-called main-belt comets (MBCs), which have orbital properties that are indistinguishable from standard asteroids with a Tisserand parameter with respect to Jupiter that is greater than three, and they display cometary activity in the form of a dust tail during part of their orbit. Numerical simulations have shown that these objects are not comets from the Kuiper belt or Oort cloud that have been recently transferred to orbits within the main belt, but instead are most likely formed in situ at their current locations (Fernández et al. 2002).

\footnotetext{
* Herschel is an ESA space observatory with science instruments provided by European-led Principal Investigator consortia and with important participation from NASA.

$\star \star$ Current address: department of Astrophysical Sciences, Princeton University, NJ 08544, USA. e-mail: valborro@princeton. edu
}

Theoretical models suggest that the snow line was initially close to the Mars orbit due to the absorption of stellar radiation by dust (Sasselov \& Lecar 2000; Lecar et al. 2006). Therefore, objects formed at their current locations in the outer asteroid belt may have been able to accumulate water ice in subsurface reservoirs, despite the effect of solar radiation. Determining the composition of this class of objects can provide important clues to both the thermal properties that allow water to survive in subsurface layers and the distribution of volatile materials in the solar nebula to constrain planet formation mechanisms. Additionally, MBCs may have played an important role in the delivery of water and other volatiles to the inner solar system, including the Earth.

The MBC 176P/LINEAR (hereafter 176P) was discovered in 1999 and originally categorized as asteroid 118401 LINEAR. This object belongs to the Themis asteroid family. Cometary activity was reported for this object around perihelion in 2005 (Hsieh et al. 2011) by the Hawaii Trails project (HTP; Hsieh \& Jewitt 2006; Hsieh 2009). It displayed a mean photometric excess of $\sim 30 \%$ during a month-long active period around its perihelion passage, consistent with an approximate total dust mass-loss of $\sim 7 \times 10^{4} \mathrm{~kg}$ (Hsieh et al. 2011). Although ice sublimation is expected to trigger MBC activity, gas emission has never been directly detected in these objects owing to their low activity, which requires very sensitive observations. Herschel proves to be the most sensitive instrument for directly observ- 
ing water in a distant comet (e.g. Bockelée-Morvan et al. 2010). In this paper we present the Herschel observation of the $1_{10}-1_{01}$ fundamental rotational transition of $\mathrm{H}_{2} \mathrm{O}$ at $557 \mathrm{GHz}$ in $176 \mathrm{P}$. This observation is intended to test the prediction that the observed cometary activity of MBCs is driven by sublimation of water ices and to constrain the production process.

\section{Observations}

The MBC 176P was observed with the Heterodyne Instrument for the Far Infrared (HIFI; de Graauw et al. 2010), one of the three instruments onboard the ESA Herschel Space Observatory (Pilbratt et al. 2010), within the framework of the Herschel guaranteed-time key program "Water and related chemistry in the solar system" (Hartogh et al. 2009). HIFI provides very high-resolution spectroscopy that can resolve the line shape and enable the determination of accurate production rates (e.g., Hartogh et al. 2010). 176P was the best MBC target in terms of its visibility close to the perihelion passage and anticipated line strength to be observed by Herschel during the mission lifetime. It passed its perihelion on 30 June 2011 at a distance of $2.57 \mathrm{AU}$ from the Sun and was observed by Herschel on UT 8.78 August 2011 with a total on-target integration time of $4.8 \mathrm{~h}$, when it was at a heliocentric distance of $2.58 \mathrm{AU}$ and a distance of $2.55 \mathrm{AU}$ from the satellite (Herschel ObsID 1342225905). The object was tracked using an up-to-date ephemeris provided by the JPL's Horizons system.

The line emission from the fundamental $\left(1_{10}-1_{01}\right)$ rotational transition of ortho-water at $557 \mathrm{GHz}$ was searched for in the upper sideband of the HIFI band 1a mixer. The observation was performed in the frequency-switching observing mode with a frequency throw of $94.5 \mathrm{MHz}$, using both the wide band spectrometer (WBS) and the high-resolution spectrometer (HRS). In this observing mode there is no need to observe a reference position on the sky and the on-target integration time is maximized. However, the statistical noise may be underestimated for observations in frequency-switched mode owing to uncertainties in baseline removal (Bockelée-Morvan et al. 2012). The spectral resolution of the WBS is $1 \mathrm{MHz}\left(\sim 0.54 \mathrm{~km} \mathrm{~s}^{-1}\right.$ at the frequency of the observed line), while the HRS was used in its high-resolution mode with a resolution of $120 \mathrm{kHz}$ $\left(\sim 0.065 \mathrm{~km} \mathrm{~s}^{-1}\right)$. The main beam brightness temperature scale was computed using a beam efficiency of 0.75 and a forward efficiency of 0.96 . The folded spectrum was obtained by averaging the original spectrum with a shifted and inverted copy. Horizontal and vertical polarizations were averaged, weighted by the root mean square amplitude, to increase the signal-tonoise ratio. The pointing offset of horizontal and vertical polarization spectra is 6.' 6 in band 1a, corresponding to approximately $20 \%$ of the half-power beam width at the observed frequency.

\section{Data analysis}

The data analysis was performed using the Herschel interactive processing environment (HIPE) software package (Ott 2010). The standard HIFI processing pipeline v7.1.0 was used to reduce the data to calibrated level-2 data products. The frequencyswitching observing mode introduces a strong baseline ripple. To obtain a reliable estimate of the noise present in the measured data, the baseline has to be removed, which is usually accomplished by fitting a linear combination of sine waves using the Lomb-Scargle periodogram technique. Nevertheless, the instrumental processes responsible for the baseline are in general combinations of linear distortions of different components in the receiver/spectrometer subsystems with a small fraction of nonlinear processes, which may cause an aperiodicity in the ripple. Analyzing such contaminated signals by assuming a linear relation among the signal components (a fundamental assumption for all the Fourier-based techniques) is not always suitable, depending on the degree of nonlinearity. In this work, we utilize a relatively novel approach specifically developed for analysis of aperiodic and nonlinear signals - the Hilbert-Huang Transform (HHT; Huang et al. 1998, 1999; Battista et al. 2007). This approach combines the empirical mode decomposition (EMD) procedure, which decomposes the original signal into its intrinsic mode functions (IMFs; representing the different modes of oscillations) with the Hilbert transform that can be then used in computing the instantaneous frequencies. The EMD technique extracts all the oscillatory modes, including all the baseline ripple components (the smooth low-frequency modes), as well as the highest frequency components, usually the noise. Another important property of IMFs is that they obey a simple additive rule to reconstruct the original signal exactly. This makes the EMD approach an accurate and versatile tool that has been successfully used in many areas from atmospheric science to cosmology such as denoising and detrending, as well as the time series analysis tool for identifying periodic and quasi-periodic features (see Duffy 2004; Battista et al. 2007, and references therein).

In this work we applied the EMD technique to the measured WBS and HRS spectra to obtain the highest frequency IMFs (dominated by Gaussian noise), which are usually the first modes (Duffy 2004). Some of the modes obtained from the decomposition of the HRS spectrum are shown in the lower panels of Fig. 1. Then, the root mean square (rms) noise of the brightness temperature in each spectrum is used to derive a 3- $\sigma$ upper limit for the $\mathrm{H}_{2} \mathrm{O}$ production rate shown in Table 1 . The brightness temperature rms of the WBS and HRS spectra differ owing to their different spectral resolutions as predicted by the Herschel observation planning tool (Hspot). The rms agrees with the value derived using the Lomb-Scargle periodogram method to determine the frequencies of the baseline. Typically the Lomb-Scargle implementation requires 20 to 30 sinusoidal components to achieve a good fit of the baseline ripple, while the HHT analysis provides a good estimate of the noise with eight modes and has an additional advantage in computing speed. Fitting a narrow frequency range of $\sim 40 \mathrm{MHz}$ around the water line with a high-order polynomial tends to underestimate the noise level determined from the whole band by about a factor of two. However, the difference introduced by the baseline fitting method is smaller than the uncertainty in the upper limit for the outgassing rate due to unknown model parameters, and therefore our data reduction method does not modify the conclusions. The EMD and HHT reduction methods applied to the baseline removal and denoising of the Herschel/HIFI data will be described in detail in a future work (Rezac et al., in prep.). We show the baseline subtracted HRS spectrum in Fig. 2 with the expected line emission overplotted.

\section{Outgassing rate}

There is no evidence of $\mathrm{H}_{2} \mathrm{O}$ emission in our observation, although it is expected that the object's dust emission activity is driven by the sublimation of subsurface material as it approaches perihelion. A molecular excitation model based on the publicly available accelerated Monte Carlo radiative transfer code ratran (Hogerheijde \& van der Tak 2000) is used to calculate the population of the rotational levels of water as a 


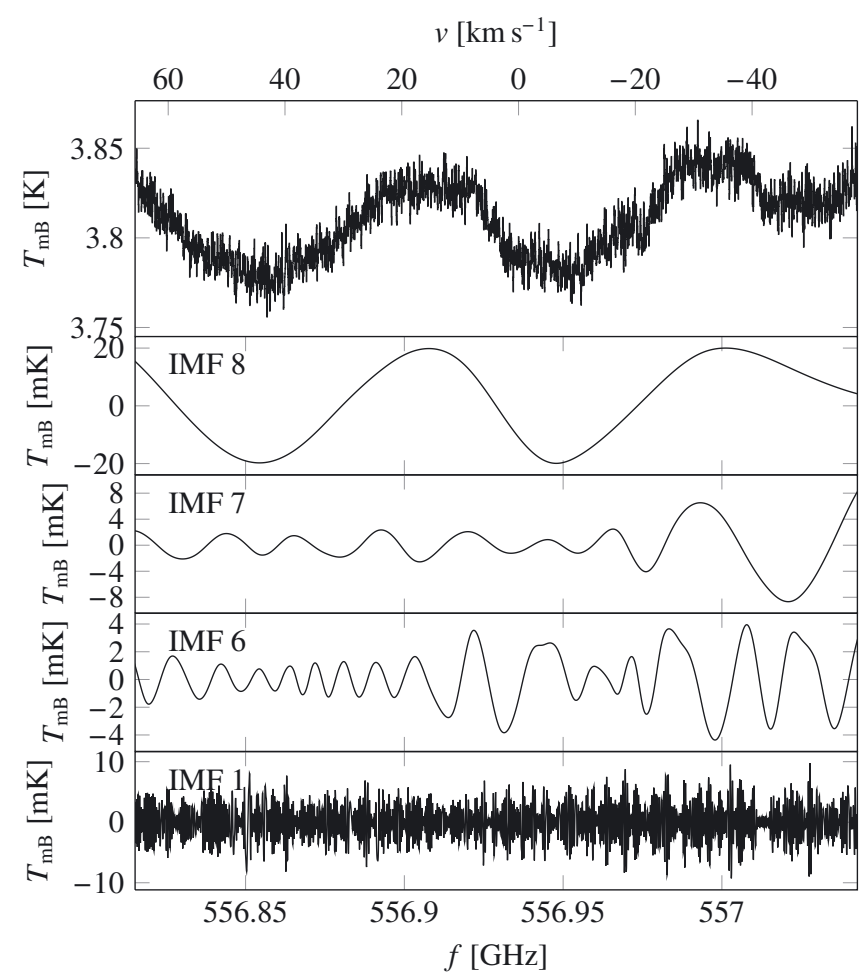

Fig. 1. Original HRS subband 1 spectrum of the $\mathrm{H}_{2} \mathrm{O} 1_{10}-1_{01}$ line at $556.936 \mathrm{GHz}$ observed on UT 8.78 August (upper panel), and several low and high frequency components of the spectrum determined using the EMD analysis (four lower panels) with labels indicating the mode number. The vertical axis is the calibrated main beam brightness temperature. The lower horizontal axis is the upper sideband frequency, while the upper axis shows the velocity with respect to the nucleus's rest frame.

Table 1. Standard deviation of the brightness temperature and line area, and retrieved 3- $\sigma$ upper limits of the $\mathrm{H}_{2} \mathrm{O}$ production rate in 176P/LINEAR.

\begin{tabular}{lccc}
\hline \hline Spectrometer & $\begin{array}{c}\sigma_{T_{\mathrm{mB}}} \\
(\mathrm{K})\end{array}$ & $\begin{array}{c}\sigma_{\int T_{\mathrm{mB}} \mathrm{d} v} \\
\left(\mathrm{~K} \mathrm{~km} \mathrm{~s}^{-1}\right)\end{array}$ & $\begin{array}{c}Q_{\mathrm{H}_{2} \mathrm{O}}{ }^{a} \\
\left(\mathrm{~mol} \mathrm{~s}^{-1}\right)\end{array}$ \\
\hline WBS & $6.598 \times 10^{-4}$ & $6.172 \times 10^{-4}$ & $<2.08 \times 10^{25}$ \\
HRS & $1.998 \times 10^{-3}$ & $6.365 \times 10^{-4}$ & $<2.14 \times 10^{25}$ \\
\hline
\end{tabular}

Notes. ${ }^{(a)}$ Production rates derived for a gas kinetic temperature of $20 \mathrm{~K}$, expansion velocity of $0.5 \mathrm{~km} \mathrm{~s}^{-1}$, and an electron density scaling factor of $x_{n_{\mathrm{e}}}=0.2$.

function of the nucleocentric distance. The code includes collisional effects and infrared fluorescence by solar radiation to derive the production rates. We used the one-dimensional spherically symmetric version of the code following the description outlined in Bensch \& Bergin (2004) that has been used to analyze Herschel and ground-based cometary observations (see e.g. Hartogh et al. 2010, 2011; de Val-Borro et al. 2010, 2012). The model input parameters are the gas kinetic temperature, which controls the molecular excitation in the collisional region, and the electron density. We assume a gas kinetic temperature in the range $20-40 \mathrm{~K}$. The electron density and temperature profiles from Biver (1997) are adopted. Since the electron density in the coma is not well constrained, an electron density scaling factor of $x_{n_{\mathrm{e}}}=0.2$ with respect to the standard profile derived from observations of comet $1 \mathrm{P} /$ Halley has been used (e.g. Hartogh et al. 2010). The expansion velocity is assumed to be

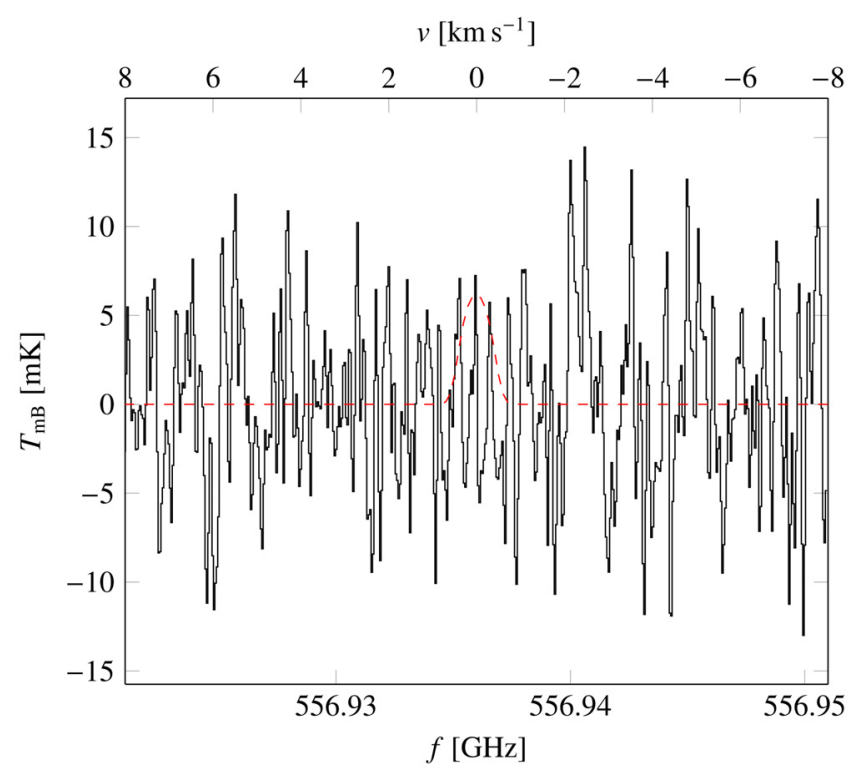

Fig. 2. High-frequency component of the HRS spectrum of the $\mathrm{H}_{2} \mathrm{O} 1_{10}-1_{01}$ line at $556.936 \mathrm{GHz}$ observed on UT 8.78 August with overplotted synthetic spectrum of the 3- $\sigma$ upper limit shown as the dashed line. The vertical axis is the calibrated main beam brightness temperature. The lower horizontal axis is the upper sideband frequency, while the upper axis shows the velocity with respect to the nucleus's rest frame.

constant in the coma, and the radial gas density profile for water was obtained using the standard spherically symmetric Haser distribution. The gas expansion velocity derived from the preand post-perihelion evolution of comet C/1995 O1 (Hale-Bopp) is given by $v_{\exp }=1.12 \times r_{\mathrm{h}}^{-0.41} \mathrm{~km} \mathrm{~s}^{-1}$ (Biver et al. 1997), which corresponds to $0.76 \mathrm{~km} \mathrm{~s}^{-1}$ at 176P's heliocentric distance. Since Hale-Bopp was a very active comet, this expansion velocity is a very conservative upper limit. A thermal velocity of $0.35 \mathrm{~km} \mathrm{~s}^{-1}$ is obtained from the temperature expected at the subsolar point where ice sublimates. Then, as cometary atmospheres are formed by quasi-adiabatic expansion, the gas accelerates as it expands, as observed with the large field of view of the $18-\mathrm{cm} \mathrm{OH}$ observations. For low-activity and distant comets an expansion velocity close to $0.5 \mathrm{~km} \mathrm{~s}^{-1}$ is determined from the shapes of the $\mathrm{OH}$ line observed with the Nançay radio telescope (Tseng et al. 2007), but observations for $Q_{\mathrm{OH}}<10^{28} \mathrm{~mol} \mathrm{~s}^{-1}$ are lacking, and observations at $r_{\mathrm{h}}>2 \mathrm{AU}$ are rare. Odin observations of the $\mathrm{H}_{2} \mathrm{O} 557 \mathrm{GHz}$ line toward the active comet $\mathrm{C} / 2003$ K4 (LINEAR) at 2.2 AU from the Sun are consistent with an expansion velocity on the order of $0.5 \mathrm{~km} \mathrm{~s}^{-1}$ (Biver et al. 2007). The dust particles ejected by $176 \mathrm{P}$ have an approximate velocity or $\sim 5 \mathrm{~m} \mathrm{~s}^{-1}$ calculated from numerical simulations to match optical observations (Hsieh et al. 2011). However, dust is expected to be much slower than gas if it is formed by large and heavy particles with very low outgassing rates.

The mean of the derived upper limits on the total $\mathrm{H}_{2} \mathrm{O}$ column density integrated within the beam for the considered model parameters is $\left\langle N_{\mathrm{H}_{2} \mathrm{O}}\right\rangle<3 \times 10^{10} \mathrm{~cm}^{-2}$. For a low expansion velocity characteristic of weak comets, we derive a sensitive 3- $\sigma$ upper limit on the water production rate of $<2.1 \times 10^{25} \mathrm{~mol} \mathrm{~s}^{-1}$ from the WBS and HRS data (see Table 1). An upper limit of $<4 \times 10^{25} \mathrm{~mol} \mathrm{~s}^{-1}$ is derived from the mean of the WBS and HRS upper limits for gas expansion velocities between $0.4-0.7 \mathrm{~km} \mathrm{~s}^{-1}$ and gas kinetic temperatures between $20-40 \mathrm{~K}$. 
With the exception of 133P/Elst-Pizarro, our upper limit for the $\mathrm{H}_{2} \mathrm{O}$ production rate is more stringent than any of those derived in other $\mathrm{MBC}$ s from the spectroscopic search for $\mathrm{CN}$ emission in the optical when the objects were active. In addition, it does not require the uncertain assumption of a $Q_{\mathrm{CN}} / Q_{\mathrm{H}_{2} \mathrm{O}}$ value. Adopting a $Q_{\mathrm{CN}} / Q_{\mathrm{H}_{2} \mathrm{O}}$ mixing ratio of $10^{-3}$ the upper limits are $Q_{\mathrm{H}_{2} \mathrm{O}}<1.3 \times 10^{24} \mathrm{~mol} \mathrm{~s}^{-1}$ in 133P/Elst-Pizarro (Licandro et al. 2011), $Q_{\mathrm{H}_{2} \mathrm{O}}<1.4 \times 10^{26} \mathrm{~mol} \mathrm{~s}^{-1}$ in $\mathrm{P} / 2008 \mathrm{R} 1$ (Garradd) (Jewitt et al. 2009), $Q_{\mathrm{H}_{2} \mathrm{O}}<1.3 \times 10^{27} \mathrm{~mol} \mathrm{~s}^{-1}$ in P/2006 VW139 (Hsieh et al. 2012b), $Q_{\mathrm{H}_{2} \mathrm{O}}<6 \times 10^{26} \mathrm{~mol} \mathrm{~s}^{-1}$ in P/2010 R2 (La Sagra) (Hsieh et al. 2012c), and $Q_{\mathrm{H}_{2} \mathrm{O}}<9 \times 10^{26} \mathrm{~mol} \mathrm{~s}^{-1}$ in the collisionally disrupted main belt object (596) Scheila (Hsieh et al. 2012a). However, no direct search for $\mathrm{H}_{2} \mathrm{O}$ has been carried out before, and there are substantial uncertainties in the estimation of the water production from the $\mathrm{CN}$ emission given the wide range of observed $Q_{\mathrm{CN}} / Q_{\mathrm{H}_{2} \mathrm{O}}$ ratios in comets and their dependence on heliocentric distance. Therefore, these previously published values can only be considered as order-of-magnitude approximations.

\section{Discussion}

We observed water emission in $176 \mathrm{P}$ with Herschel/HIFI to test the prediction that cometary activity in MBCs is driven by sublimation of water ices from the nucleus. There are several mechanisms that have been proposed to drive mass loss from small bodies, including sublimation of subsurface ices, rotational instability, impact ejection and thermal fracture (see Bertini 2011; Jewitt 2012, for recent reviews of MBC physical properties and activation mechanisms). The cometary activity observed in $176 \mathrm{P}$ was initially found to suggest the presence of sublimating subsurface ice that may have been exposed by recent collisions (Hsieh et al. 2011). This view is supported by the detection of water ice absorption in spectroscopic observations centered at $3.1 \mu \mathrm{m}$ of the surface of the largest asteroid of the Themis asteroid family, 24 Themis, which belongs to the same dynamical family as 176P (Rivkin \& Emery 2010; Campins et al. 2010), although it has been claimed that the measured spectra are consistent with the transmission spectra of goethite (Beck et al. 2011). From the search for the $\mathrm{H}_{2} \mathrm{O} 1_{10}-1_{01}$ rotational line at $557 \mathrm{GHz}$ in 176P, a 3- $\sigma$ upper limit for the $\mathrm{H}_{2} \mathrm{O}$ production rate of $<4 \times 10^{25} \mathrm{~mol} \mathrm{~s}^{-1}$ is derived from the WBS and HRS data, for gas expansion velocities between $0.4-0.7 \mathrm{~km} \mathrm{~s}^{-1}$ and gas kinetic temperatures between 20 and $40 \mathrm{~K}$. Using the peak value of the $R$-band magnitude, $m(1,1,0)=15.35 \pm 0.05$, measured when the object was active in late 2005 at a heliocentric distance of 2.58 AU, a $V$-band magnitude of $m_{V}\left(1, r_{\mathrm{h}}, 0\right)=17.8$ is obtained (Hsieh et al. 2009). Since the cometary activity in $176 \mathrm{P}$ is indicative of ice sublimation with a $30 \%$ contribution of the coma to the total brightness, the scaling relation between gas production rates and heliocentric magnitudes from Jorda et al. (2008) predicts a water production rate of approximately $1.0 \times 10^{26} \mathrm{~mol} \mathrm{~s}^{-1}$. This correlation has been obtained for a sample of 37 comets with heliocentric distances between 0.32-4.53 AU. Thus, if ice sublimation is the driving mechanism of 176P's activity, the derived $\mathrm{H}_{2} \mathrm{O}$ production rate is too low by about a factor of two to explain the activity level during its 2005 perihelion passage. It is unlikely that sublimation of carbon monoxide ice is the source of the activity in $\mathrm{MBCs}$ since the temperature in the region where the comet formed could not have allowed condensation of CO. We note that the Jorda et al. (2008) correlation should be taken with some care because none of the comet measurements were obtained at the brightness level of 176P. From the dust production rate of $0.07 \mathrm{~kg} \mathrm{~s}^{-1}$ estimated by Hsieh et al. (2011), a water production rate of $2.3 \times 10^{24} \mathrm{~mol} \mathrm{~s}^{-1}$ is inferred assuming a dustto-gas ratio of one. However, there are significant uncertainties in the value of the dust production rate determined from photometric data, and additionally the dependence on the dust-togas ratio with heliocentric distance is poorly constrained. Midinfrared photometric observations of 176P on 23-24 April 2010 by the Wide-field Infrared Survey Explorer (WISE) mission did not provide any indication of a coma (Bauer et al. 2012), and there are no other published infrared or optical data closer to the last perihelion passage. We conclude that water was not detected in our observation because the water production rate was lower than $4 \times 10^{25} \mathrm{~mol} \mathrm{~s}^{-1}$ or the object was not active during our observation, so a more detailed study is needed to shed light on the activation mechanism in MBCs.

Acknowledgements. HIFI has been designed and built by a consortium of institutes and university departments from across Europe, Canada, and the United States under the leadership of SRON, Netherlands Institute for Space Research, Groningen, The Netherlands, and with major contributions from Germany, France, and the US. HIPE is a joint development by the Herschel Science Ground Segment Consortium, consisting of ESA, the NASA Herschel Science Center, and the HIFI, PACS, and SPIRE consortia. Support for this work was provided by NASA through an award issued by JPL/Caltech. M.d.V.B. was supported by the Special Priority Program 1488 of the German Science Foundation. S.S. was supported by Polish MNiSW funds (181/N-HSO/2008/0). We acknowledge the referee, $\mathrm{H}$. Campins, for his comments.

\section{References}

Battista, B. M., Knapp, C., McGee, T., \& Goebel, V. 2007, Geophysics, 72, H29 Bauer, J. M., Mainzer, A. K., Grav, T., et al. 2012, ApJ, 747, 49

Beck, P., Quirico, E., Sevestre, D., et al. 2011, A\&A, 526, A85

Bensch, F., \& Bergin, E. A. 2004, ApJ, 615, 531

Bertini, I. 2011, Planet. Space Sci., 59, 365

Biver, N. 1997, Ph.D. Thesis, Univ. Paris 7-Diderot

Biver, N., Bockelée-Morvan, D., Colom, P., et al. 1997, Earth Moon and Planets, 78,5

Biver, N., Bockelée-Morvan, D., Crovisier, J., et al. 2007, Planet. Space Sci., 55, 1058

Bockelée-Morvan, D., Biver, N., Crovisier, J., et al. 2010, in AAS/Division for Planetary Sciences Meeting Abstracts, BAAS, 42, 946

Bockelée-Morvan, D., Biver, N., Swinyard, B., et al. 2012, A\&A, 544, L15

Campins, H., Hargrove, K., Pinilla-Alonso, N., et al. 2010, Nature, 464, 1320 de Graauw, T., Helmich, F. P., Phillips, T. G., et al. 2010, A\&A, 518, L6 de Val-Borro, M., Hartogh, P., Crovisier, J., et al. 2010, A\&A, 521, L50 de Val-Borro, M., Hartogh, P., Jarchow, C., et al. 2012, A\&A, 545, A2 Duffy, D. G. 2004, J. Atmos. Ocean. Technol., 21, 599

Fernández, J. A., Gallardo, T., \& Brunini, A. 2002, Icarus, 159, 358

Hartogh, P., Lellouch, E., Crovisier, J., et al. 2009, Planet. Space Sci., 57, 1596

Hartogh, P., Crovisier, J., de Val-Borro, M., et al. 2010, A\&A, 518, L150

Hartogh, P., Lis, D. C., Bockelée-Morvan, D., et al. 2011, Nature, 478, 218

Hayashi, C. 1981, Prog. Theor. Phys. Suppl., 70, 35

Hogerheijde, M. R., \& van der Tak, F. F. S. 2000, A\&A, 362, 697

Hsieh, H. H. 2009, A\&A, 505, 1297

Hsieh, H. H., \& Jewitt, D. 2006, Science, 312, 561

Hsieh, H. H., Jewitt, D., \& Fernández, Y. R. 2009, ApJ, 694, L111

Hsieh, H. H., Ishiguro, M., Lacerda, P., \& Jewitt, D. 2011, AJ, 142, 29

Hsieh, H. H., Yang, B., \& Haghighipour, N. 2012a, ApJ, 744, 9

Hsieh, H. H., Yang, B., Haghighipour, N., et al. 2012b, ApJ, 748, L15

Hsieh, H. H., Yang, B., Haghighipour, N., et al. 2012c, AJ, 143, 104

Huang, N. E., Shen, Z., Long, S. R., et al. 1998, Roy. Soc. London Proc. Ser. A, 454, 903

Huang, N. E., Shen, Z., \& Long, S. 1999, Ann. Rev. Fluid Mech., 31, 417

Jewitt, D. 2012, AJ, 143, 66

Jewitt, D., Yang, B., \& Haghighipour, N. 2009, AJ, 137, 4313

Jorda, L., Crovisier, J., \& Green, D. W. E. 2008, LPI Contributions, 1405, 8046 Lecar, M., Podolak, M., Sasselov, D., \& Chiang, E. 2006, ApJ, 640, 1115

Licandro, J., Campins, H., Tozzi, G. P., et al. 2011, A\&A, 532, A65

Ott, S. 2010, in Astronomical Data Analysis Software and Systems XIX, eds.

Y. Mizumoto, K.-I. Morita, \& M. Ohishi, ASP Conf. Ser., 434, 139

Pilbratt, G. L., Riedinger, J. R., Passvogel, T., et al. 2010, A\&A, 518, L1

Rivkin, A. S., \& Emery, J. P. 2010, Nature, 464, 1322

Sasselov, D. D., \& Lecar, M. 2000, ApJ, 528, 995

Tseng, W.-L., Bockelée-Morvan, D., Crovisier, J., Colom, P., \& Ip, W.-H. 2007, A\&A, 467, 729 